\title{
Equivalent Network Approach for Field Analysis of 3-D Optical Waveguide Step Discontinuity and their Dynamic Behavior
}

\author{
Yasuhiro Hasegawa Manabu Inoue Takaharu Hiraoka Hsu, Jui-Pang \\ Faculty of Engineering, Kanagawa University, Yokohama, Japan
}

\begin{abstract}
Exact analysis of step discontinuity of 3-D optical waveguide is important for exact design of optical integrated circuit. Mode theory is introduced to derive the equivalent network in lateral direction and that in waveguide direction. The former is used to calculate waveguide propagation mode, which leads to the equivalent network along waveguide direction. The latter is used for field analysis of step discontinuity of 3-D optical waveguide. Two kinds of so far derived equivalent network are used to give reasonable analytical results with success.

Keywords ; 3-D optical waveguide step discontinuity , Equivalent network , Eigenmode , Field distribution
\end{abstract}

\section{INTRODUCTION}

Exact analysis and design of optical passive circuit such as step discontinuity shown in Fig.1 is strongly needed with advent of manufacturing technique for fine structure. This needs may be met by powerful microwave theory and network theory ${ }^{[1]}$. It is demonstrated that field problem for 3-D optical waveguide and step discontinuity shown in Fig. 1 can be treated by mode theory and equivalent network, which is very popular at microwaves. Mode theory and circuit theory is introduced to derive the optical planar circuit of TE and TM mode ${ }^{[2]}$, equivalent network in the lateral direction ${ }^{[4]}$ and that in waveguide direction. The former is used to calculate optical propagation mode, which leads to give equivalent network along waveguide direction. The latter, i.e., equivalent network along waveguide direction is used for field analysis at step discontinuity of 3-D optical waveguide.

3-D optical waveguide treated here is strip loaded guide as shown in Fig.1. In order to avoid continuous spectrum of radiation mode for open structure, rectangular waveguide-type enclosure (solid line) is provided to cover 3-D optical waveguide as shown in Fig.1, which can make continuous spectrum to discrete spectrum. At first waveguide propagation mode of optical waveguide is calculated by lateral equivalent network $^{[4]}$. Then, network parameters of equivalent network along waveguide direction is calculated for uniform 3-D optical waveguide; also equivalent network for step discontinuity is given by ideal transformer bank, whose transformer ratio is calculated by overlapping integral of the related waveguide propagation mode. Based upon this equivalent network along waveguide direction we can calculate the excited amplitude of propagation mode at both side of the step discontinuity for any mode incidence. The essential problem through these analysis is how many slab-mode must be taken into consideration for exact calculation of waveguide propagation mode and how many waveguide propagationmode for exact field analysis at the step discontinuity. These problems are investigated by convergence of the continuity of tangential field component and/or related physical parameters with mode. So far mentioned equivalent network approach is used with success.

\section{CALCULATION OF PROPAGATION EIGENMODE}

Propagation eigenmodes( mode effective refractive index and mode fielddistribution) for strip loaded 3-D optical waveguide shown in Fig.2(a) are calculated based on lateral equivalent network given in Fig.2(c), whose detail theory including how to calculate network parameters of the equivalent network are already presented in our another paper ${ }^{[4]}$. Hence calculated results only are explained here. Dimension and mate-

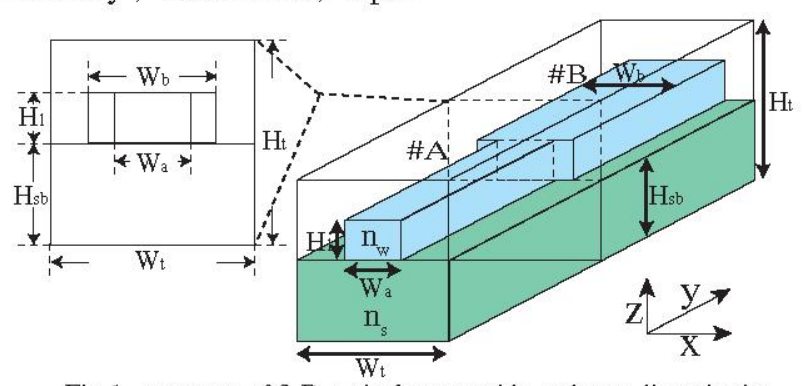

Fig.1 structure of 3-D optical waveguide and step dicontinuity

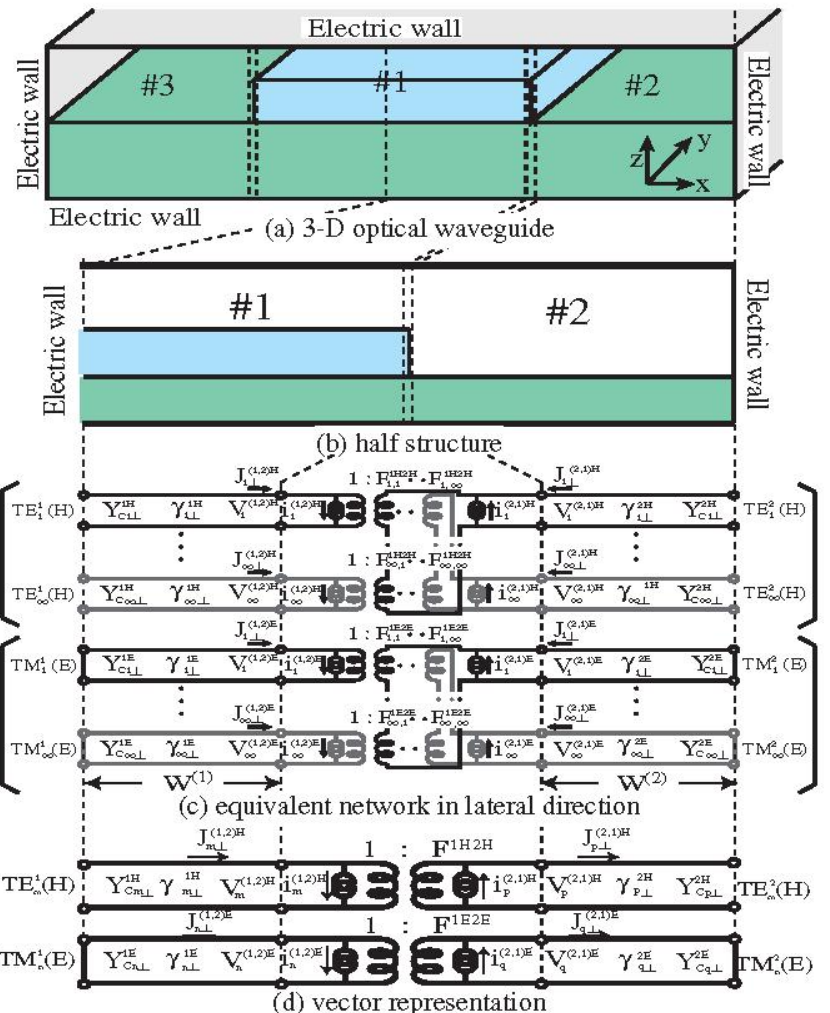

Fig.2 3-D optical waveguide structure, equivalent network in lateral direction for half structure and vector representation

rial constant are following : film thickness $\mathrm{Hl}=1[\mu \mathrm{m}]$ and its refractive index $n_{n}=1.49$, substrate thickness $\mathrm{Hsb}=2[\mu \mathrm{m}]$ and its refractive index $\mathrm{n}_{\mathrm{s}}=1.47$. This optical waveguide is covered by $4[\mu \mathrm{m}] \times 4[\mu \mathrm{m}]$ rectangular waveguide as shown in Fig.1. Through the analysis electric wall symmetry at the middle of the waveguide is assumed. So TE-like mode with electric symmetry is calculated. Calculated results of mode effective refractive index up to about 200 modes are shown in Fig.5(a) as a function of strip width $\mathrm{W}$. These calculated results are classified as guided mode(true optical mode), substrate mode and air mode, whose typical field distribution of dominant components are shown in Fig.5(c). Also how to confine the field distribution of the dominant mode with stripwidth is demonstrated in Fig.5(d), where $A=1.5[\mu \mathrm{m}], \mathrm{B}=2.0[\mu \mathrm{m}]$, $\mathrm{C}=2.1[\mu \mathrm{m}], \mathrm{D}=2.3[\mu \mathrm{m}], \mathrm{E}=2.5[\mu \mathrm{m}]$ in strip-width. 


\section{CIRCUIT ANALYSIS OF FIELD AT STEP BY EQUIVALENT NETWORK ALONG WAVEGUIDE}

When mode voltage/current is defined by sum and difference of power wave as $v=a+b, i=a-b$, the corresponding equivalent network along waveguide is given by multi-transmission line as shown in Fig.3, whose characteristic impedance is unity for any mode of waveguide \#A and\#B . Coupling of propagation mode in waveguide \#A and \#B at step discontimuity is given by ideal transformer ratio, which is calculated by eq.(1)

$$
F_{n m}^{B A}=\frac{1}{S} \iint_{S}\left(\mathbf{e}_{t n}^{B}(x, z) \times \mathbf{h}_{t m}^{A}(x, z)\right) \cdot j d S
$$

Then equivalent network for whole step discontinuity is given by Fig.3(b) or (c) in vector representation, where ideal transformer bank $\mathrm{F}^{\mathrm{BA}}$ is used in matrix form. Mode voltage/current column matrix at each port is defined as $v^{A}, v^{B}, i^{A}, i^{B}$, then following relation exists.

$$
\begin{aligned}
& v^{A}=\left(F^{B A}\right)^{t} v^{B} \quad i^{B}=F^{B A} i^{A} \\
& \text { where } v^{P}=a^{P}+b^{P}, \quad i^{P}=a^{P}-b^{P} \quad(P=A, B)
\end{aligned}
$$

Scattering matrix of step discontinuity is derived by matrix operation in the following equation.

$$
\left(\begin{array}{l}
b^{A} \\
b^{B}
\end{array}\right)=\left[\begin{array}{l}
S^{A A}=(\bar{z}+I)^{-1}(\bar{z}-I), S^{A B}=2(\bar{z}+I)^{-1}\left(\mathbf{F}^{A B}\right) \\
S^{B A}=2(\bar{y}+I)^{-1} \mathbf{F}^{A B}, S^{B B}=-(\bar{y}+I)^{-1}(\bar{y}-I)
\end{array}\right]\left(\begin{array}{l}
a^{A} \\
a^{B}
\end{array}\right)
$$$$
\text { where } \quad \bar{z}=\left(\mathbf{F}^{A B}\right)^{t} \mathbf{F}^{A B} \quad \bar{y}=\mathbf{F}^{A B}\left(\mathbf{F}^{A B}\right)^{t}
$$

From eq.(3) we can calculate scattered power wave $b^{A}, b^{8}$ for incident power wave $a^{A}, a^{B}$. Then we can calculate the mode voltage $v_{m}^{A}(y), v_{n}^{B}(y)$ and mode current distribution $i_{m}^{A}(y), i_{n}^{B}(y)$ along optical waveguide $\mathrm{A}$ and $\mathrm{B}$. Then field distribution at any position is calculated by the following mode summation, where $\mathrm{p}=\mathrm{A}$ or $\mathrm{B}$.

$$
\begin{cases}\mathbf{E}_{t}^{p}(x, y, z)=\sum_{m} v_{m}^{p}(y) \cdot \mathbf{e}_{t n}^{p}(x, z), & E_{y}(x, y, z)=\sum_{m} i_{m}^{p}(y) \cdot e_{y m}(x, z) \\ \mathbf{H}_{t}^{p}(x, y, z)=\sum_{m} i_{m}^{p}(y) \cdot h_{t n}^{p}(x, z), & H_{y}(x, y, z)=\sum_{m} v_{m}^{p}(y) \cdot h_{y m}(x, z)\end{cases}
$$

\section{CALCURATED RESURTS}

Dominant mode incidence at waveguide \#A is assumed. Two step cases are treated.Case1: $1.5[\mu \mathrm{m}]$ to $2.5[\mu \mathrm{m}]$, Case $2: 2.5[\mu \mathrm{m}]$ to $1.5[\mu \mathrm{m}]$. A. Excited mode amplitudes at the step discontinuity are calculated by eq. (3) and shown in Fig.4, where power conservation is guaranteed to hold. Especially power coupling between dominant mode in \#A and \#B is about 80[\%].

$B$. Continuity of tangential field at the step with propagation mode is investigated. Four tangential field components at both side of the step discontinuity are calculated by eq.(5) and shown in Fig.6. (1b)/(1c) and (2b)/(2c) for two cases with number of propagation mode as parameter. It turns out that the smooth continuity of tangential field components at the step is realized with number of waveguide propagation mode.

C. Calculation of 2-D planar field distribution at height of [I], [ II],[ $\mathrm{II}]$ shown in Fig.6(1c)/(2c) is calculated by eq.(5) and shown in Fig.6 (1d), (1e), (1f) and(2d),(2e), (2f).

\section{CONCLUSION}

Equivalent network along waveguide is derived based on optical planar circuit equations and mode theory in order to analyze 3-D optical step discontinuity, which is given by mode corresponding multi-transmission line for uniform waveguide and ideal transformer bank for step discontinuity. Based on this equivalent network, step discontinuity of 3D optical waveguide is analyzed. It turns out that the continuity of the tangential field at step discontinuity is obtained with number of waveguide propagation mode. Also excited amplitude of reflection and transmission vs mode order is calculated, which guarantee the power conservation. Finally, 2-D planar field distribution at fixed height is calculated, whose dynamic behavior will be displayed at the site.

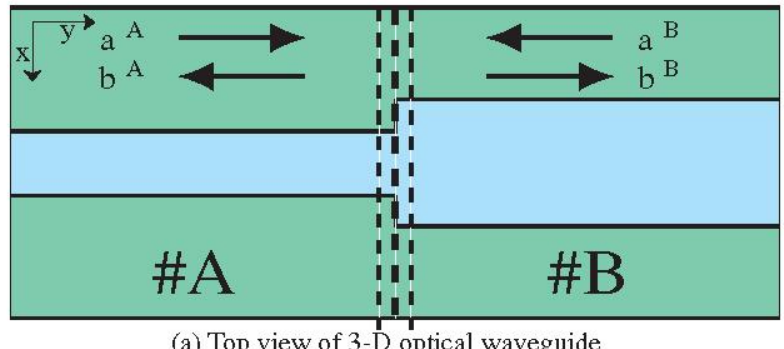

(a) Top view of 3-D optical waveguide

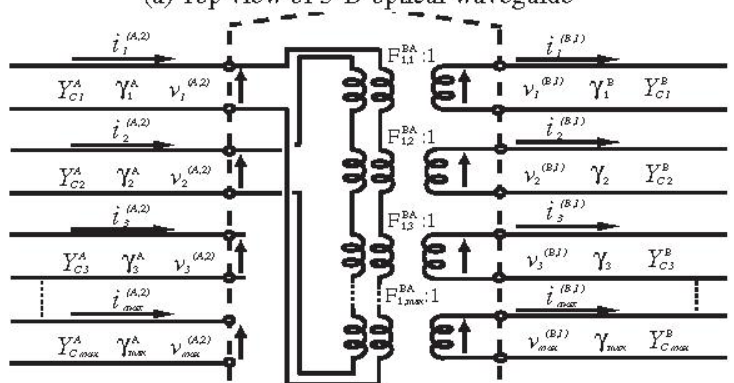

(b) equivalent network in step-discontinuity

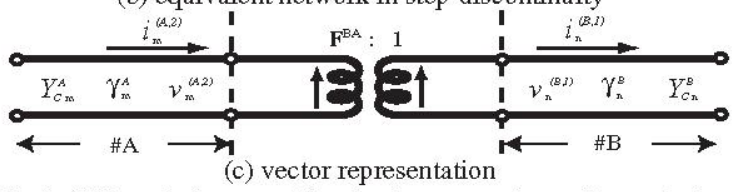

Fig.3 3-D optical waveguide of strip-type and step-discontinuity and equivalent network with vector representation

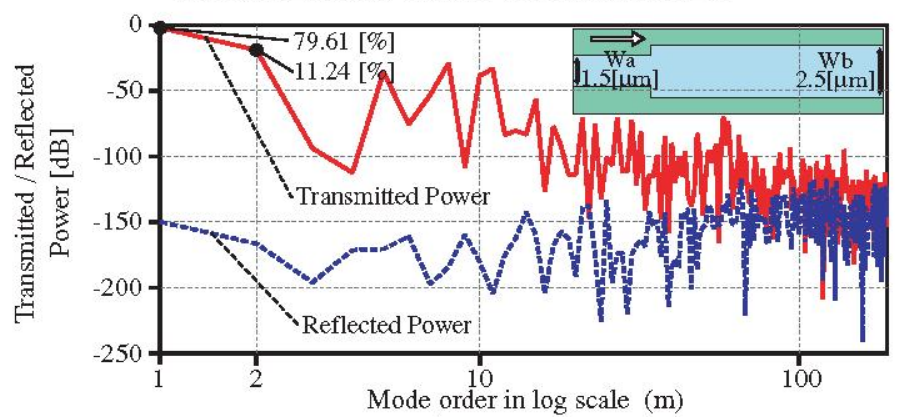

(a) Case $1(1.5[\mu \mathrm{m}]$ to $2.5[\mu \mathrm{m}])$

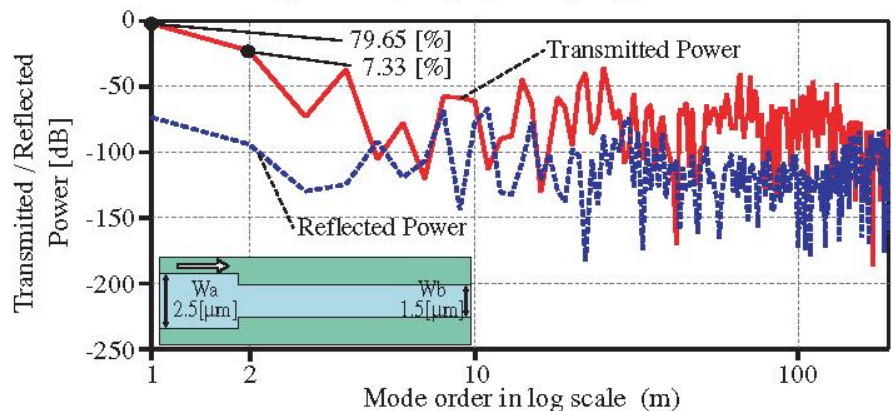

(b) Case $2(2.5[\mu \mathrm{m}]$ to $1.5[\mu \mathrm{m}])$

Fig.4 Excited mode amplitude at the step discontinuity (red solid line:transmitted power, blue dotted line:reflected power)

Reference

[1]S.T.Peng, A.A.Oliner "Guidance and Leakage properties ofaclass of open dielectric waveguides:Part1 and part2" IEEE MTT-Tranns. Vol.MTT29 pp843-855, Sep. 1981

[2]Hsu,J.P., T,Anada "Proposal of surface-Wave Planar Circuit,Formulation of its Planar Circuit Equation and Practical Application"1986 IEEE MTT- S Digest GG-4 pp797-800

[3]Hsu,J.P., T,Anada "Formulation of mode coupling equations at step discontinuity based on the planar circuit theory" 1989 IEEE MTT-S Digest PP-5 pp 1135-1138

[4]M.Inoue, T.Hiraoka, Hsu, J.P. “Analysis of Eigenmode for 3-D optical Waveguide based on Planar Circuit Equations and Lateral Equivalent Netwrok" 


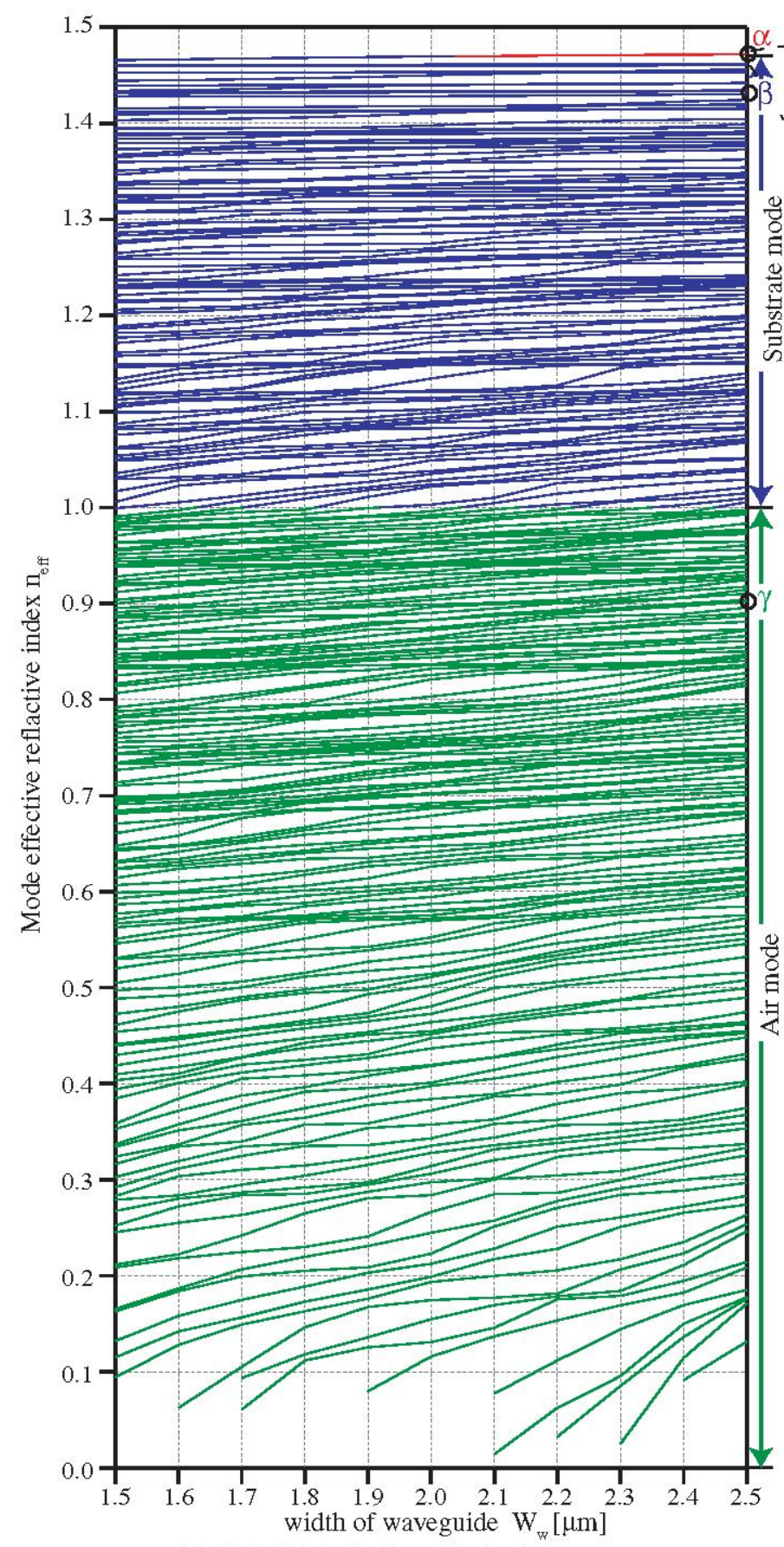

(a) Calculated effective refractive index $n_{\text {eff }}$

(red line:guided mode, blue line:substrate mode, green line:air mode)
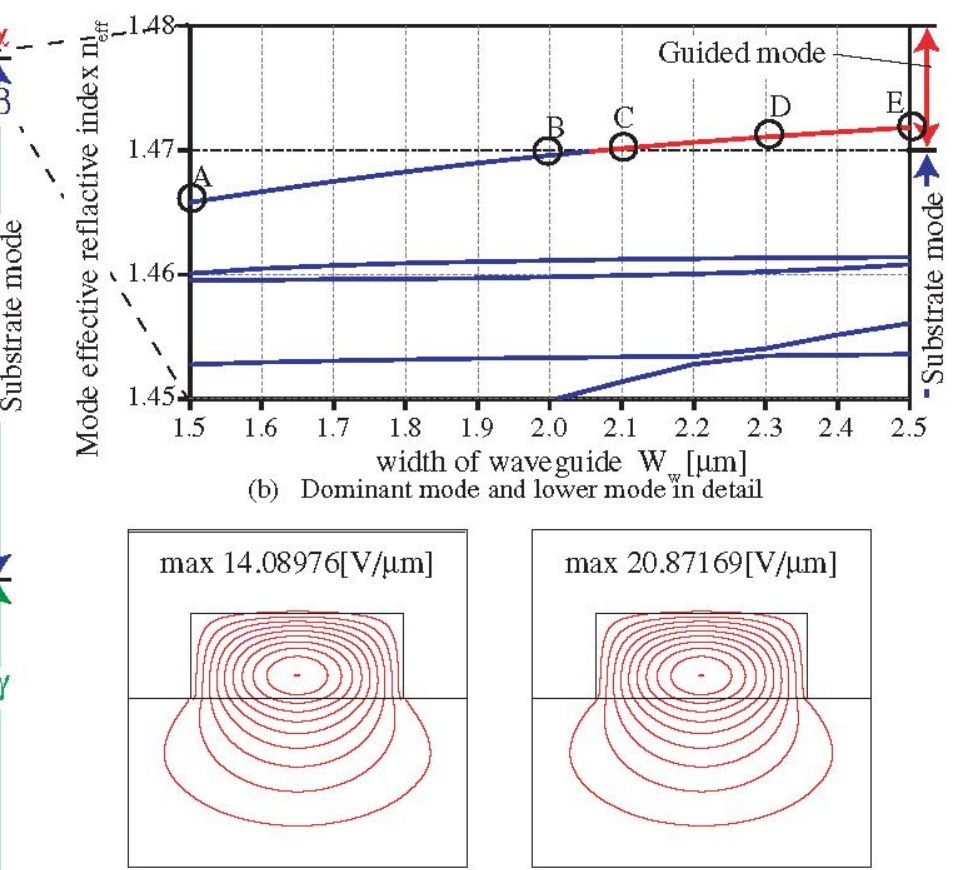

E component

(c1) Guided mode $\alpha$ (mode order $=1, \mathrm{n}_{\mathrm{eff}}=1.47182$ )

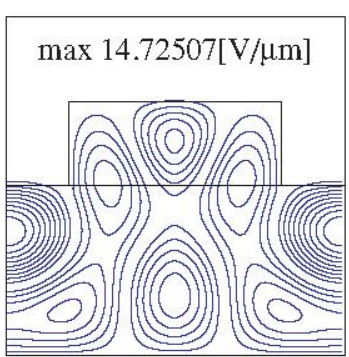

$\mathrm{E}_{\mathrm{x}}$ component $\max 20.87169[\mathrm{~V} / \mu \mathrm{m}]$

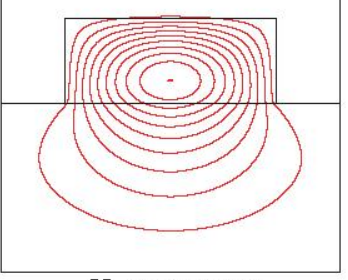

$\mathrm{H}_{z}$ component

$\max 21.54394[\mathrm{~V} / \mu \mathrm{m}]$

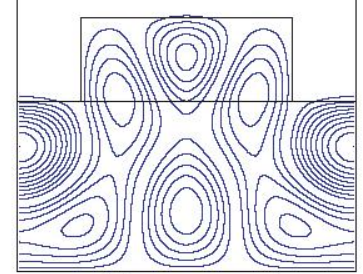

$\mathrm{H}_{\mathrm{z}}$ component

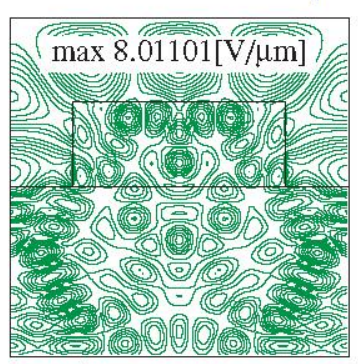

$\mathrm{E}_{\mathrm{x}}$ component

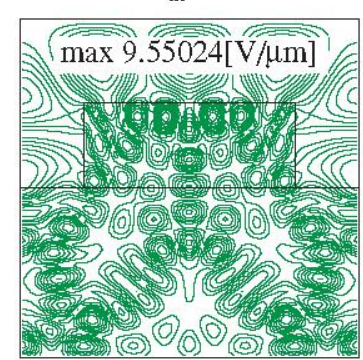

$\mathrm{H}_{\mathrm{z}}$ component

(c3) Air mode $\gamma$ (mode order $=120, \mathrm{n}=0.90367$ )

(c) Field distribution of main components ( $\left.\mathrm{W}_{\mathrm{w}}=2.5[\mu \mathrm{m}]\right)$

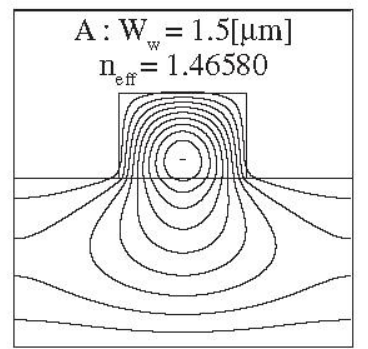

$\max 20.66284[\mathrm{~V} / \mu \mathrm{m}]$

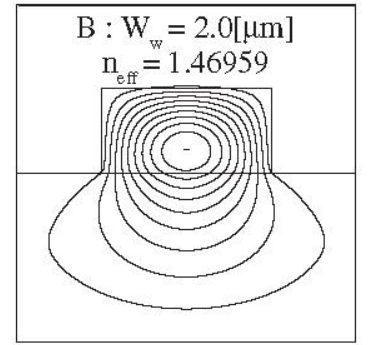

$\max 22.31186[\mathrm{~V} / \mu \mathrm{m}]$

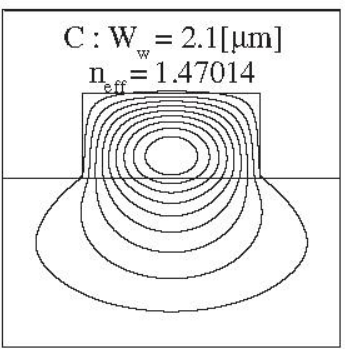

$\max 22.07654[\mathrm{~V} / \mu \mathrm{m}]$

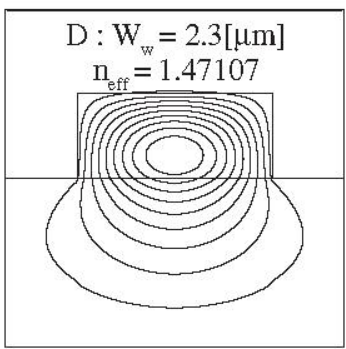

$\max 21.49792[\mathrm{~V} / \mu \mathrm{m}]$

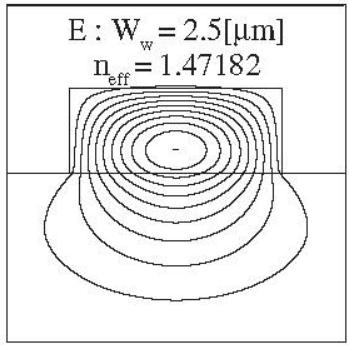

$\max 20.87169[\mathrm{~V} / \mu \mathrm{m}]$

(d) Field distribution of the dominant mode in $\mathrm{H}_{\mathrm{z}}$ for various strip-wid th Fig.5 Calculated results of eigenmode for 3-D optical waveguide 


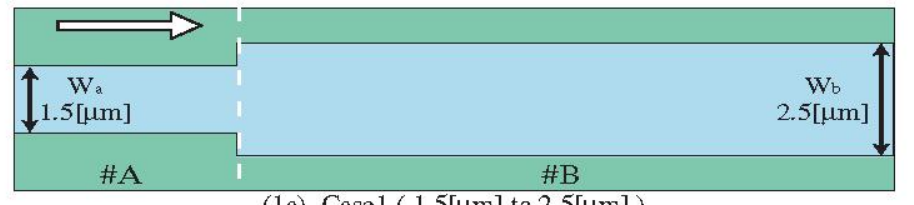

(1a) Case 1 ( $1.5[\mu \mathrm{m}]$ to $2.5[\mu \mathrm{m}])$
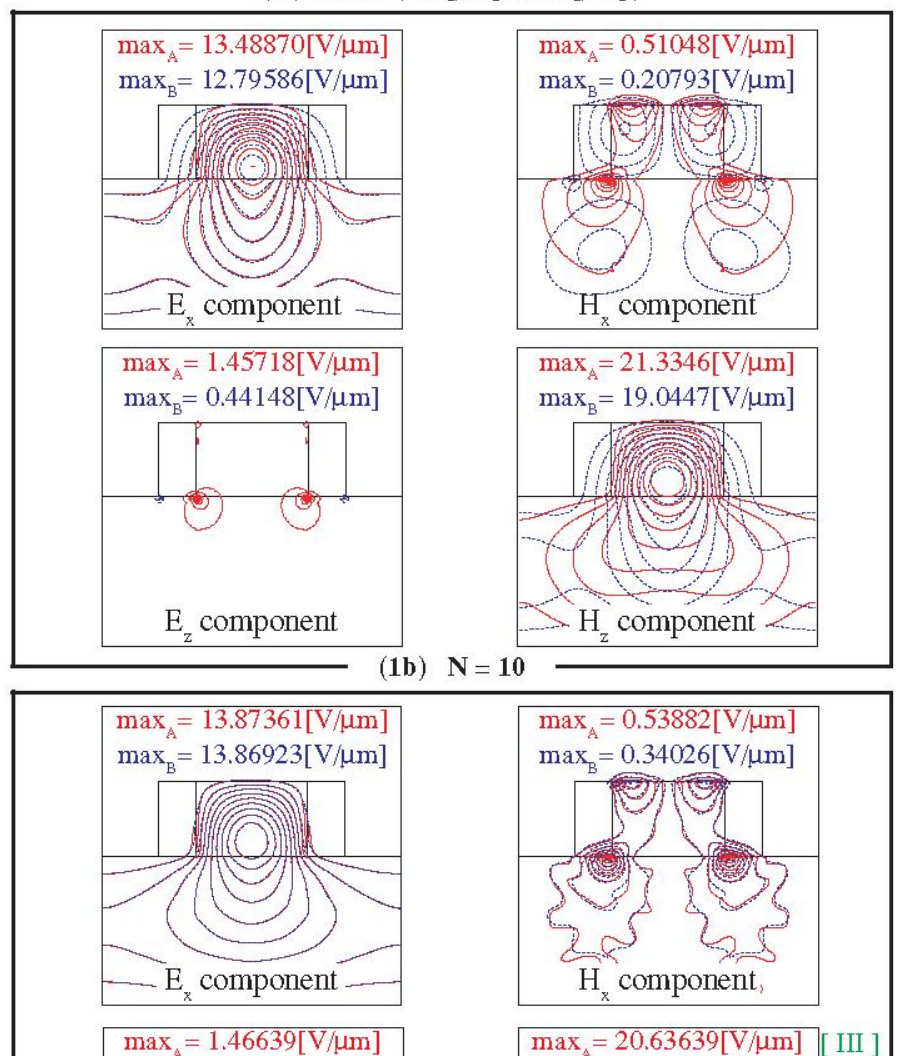

$\max _{\mathrm{B}}=0.39686[\mathrm{~V} / \mu \mathrm{m}]$

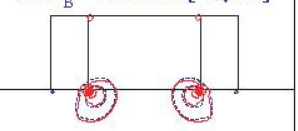

$\mathrm{E}_{\mathrm{z}}$ component

(1c) $\mathrm{N}=190$

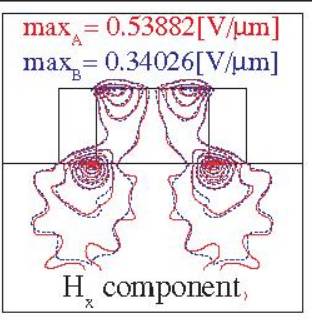

$\left.-\max _{\max _{\mathrm{A}}}=20.63639[\mathrm{~V} / \mu \mathrm{m}]\right][\mathrm{III}]$
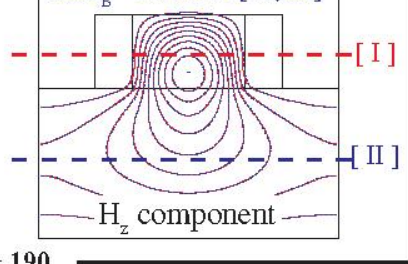

- [II ]

al field

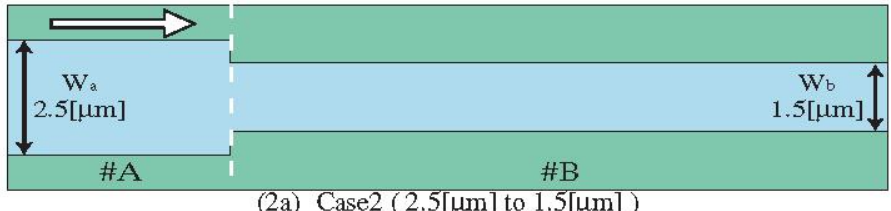

(2a) Case2 $(2.5[\mu \mathrm{m}]$ to $1.5[\mu \mathrm{m}])$
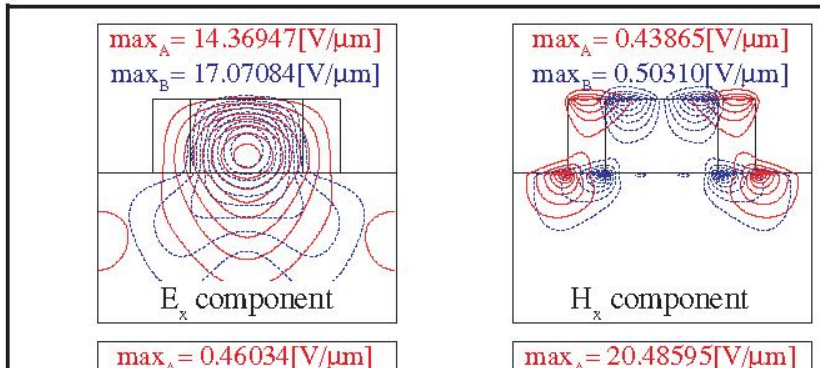

$\max _{\mathrm{B}}=1.67203[\mathrm{~V} / \mu \mathrm{m}]$

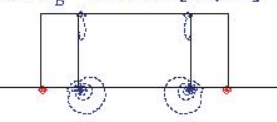

$\mathrm{E}_{\mathrm{z}}$ component

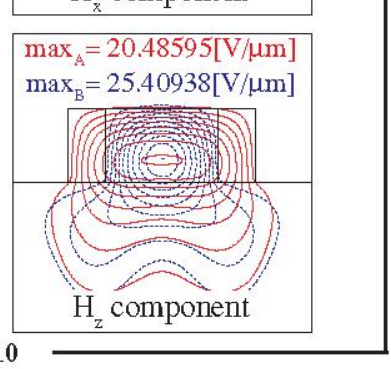

(2b)
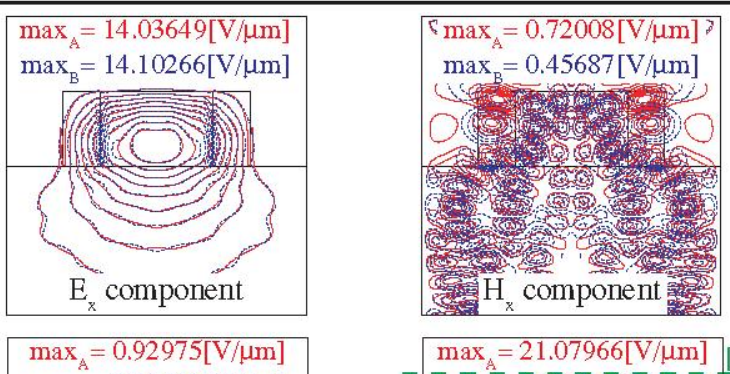

$\max _{\mathrm{B}}=3.19601[\mathrm{~V} / \mu \mathrm{m}]$

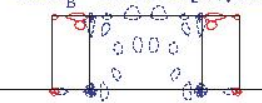

$\mathrm{E}_{\mathrm{z}}$ component

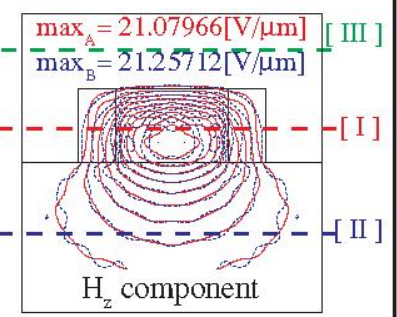

(2c) $\mathrm{N}=190$

$\mathrm{H}_{\mathrm{z}}$ component

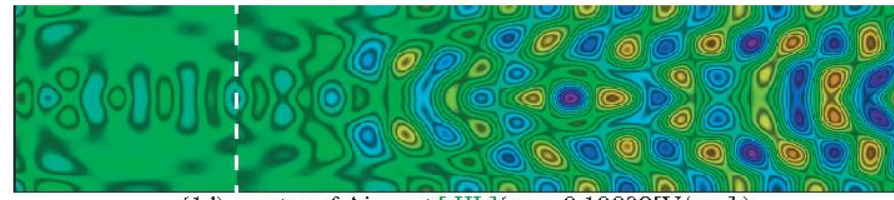

(1d) center of Air part [ III ] $(\max 0.10098[\mathrm{~V} / \mu \mathrm{m}])$

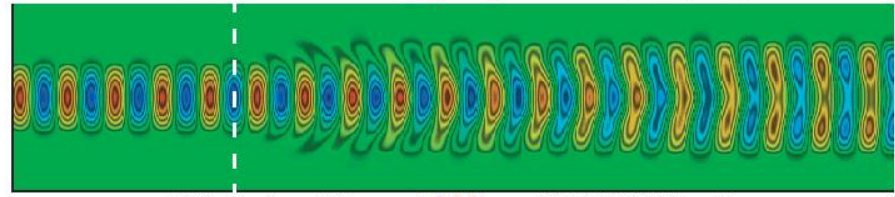

(1e) center of film part [ I ] $(\max 18.84819[\mathrm{~V} / \mu \mathrm{m}])$

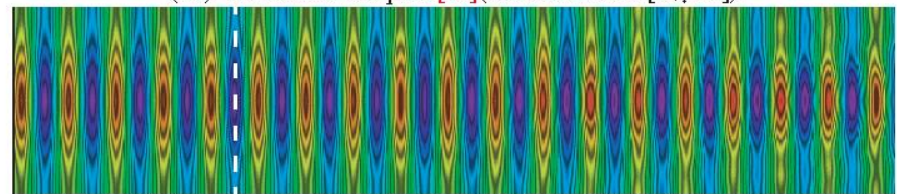

(1f) center of substrate part [ II ] $(\max 9.96661[\mathrm{~V} / \mu \mathrm{m}])$

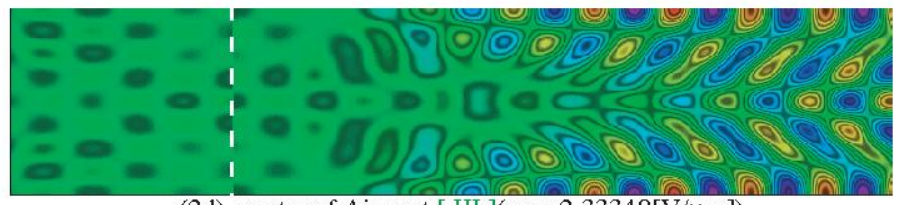

(2d) center of Air part [ III ] $(\max 2.33348[\mathrm{~V} / \mu \mathrm{m}])$

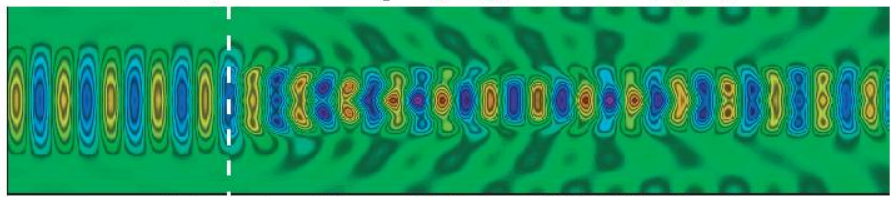

(2e) center of film part [I] $(\max 30.17381$ [V/um]

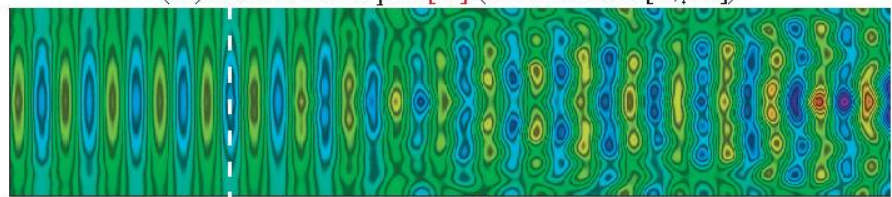

(2f) center of substrate part [ II ] $(\max 10.49972[\mathrm{~V} / \mu \mathrm{m}])$ Fig.6 Field distribution of 3-D optical waveguide at step discontinuity 